\title{
Psychological factors outmatched morphological markers in predicting limitations in activities of daily living and participation in patients with lumbar stenosis
}

\author{
V. Quack ${ }^{1 *+}$ (D) M. Boecker ${ }^{2,3+}$, C. A. Mueller ${ }^{2}$, V. Mainz ${ }^{3}$, M. Geiger ${ }^{2}$, A. W. Heinemann ${ }^{4,5}$, M. Betsch ${ }^{1}$ and \\ Y. El Mansy ${ }^{1,6}$
}

\begin{abstract}
Background: Recent demographic changes have led to a large population of older adults, many of whom experience degenerative disc diseases. Degenerative lumbar spinal stenosis (DLSS) is associated with considerable discomfort and limitations in activities of daily living (ADL). Symptomatic DLSS is one of the most frequent indications for spinal surgery. The aim of this study was to identify sociodemographic variables, morphological markers, depression as well as fear of movement that predict ADL performance and participation in social life in patients with DLSS.

Methods: Sixty-seven patients with DLSS (mean age 62.5 years [11.7], 50.7\% females) participated in the study. Predictor variables were age, gender, duration of disease, three morphological markers (severity of the lumbar stenosis, the number of affected segments and presence of spondylolisthesis) as well as self-reported depression and fear of movement. Dependent variables were pain interference with the performance of ADLs, ADLs and participation in social life. Correlations between predictor and dependent variables were calculated before stepwise, linear regression analyses. Only significant correlations were included in the linear regression analyses.

Results: Variance explained by the predictor variables ranged between $12 \%\left(R^{2}=.12\right.$; pain interference-physical) and $40 \%\left(R^{2}=.40 ; A D L\right.$ requiring lower extremity functioning; participation). Depression and fear of movement were the most powerful predictors for all dependent variables. Among the morphological markers only stenosis severity contributed to the prediction of ADLs requiring lower extremity functioning.

Conclusion: Depression and fear of movement were more important predictors of the execution of ADLs and participation in social life compared to morphological markers. Elevated depressive symptoms and fear of movement might indicate limited adaptation and coping regarding the disease and its consequences. Early monitoring of these predictors should therefore be conducted in every spine centre. Future studies should investigate whether psychological screening or a preoperative psychological consultation helps to avoid operations and enables better patient outcomes.
\end{abstract}

Keywords: Lumbar stenosis, Predictors, Morphological markers, Psychological factors, Depression, Fear of movement, Outcome

\footnotetext{
* Correspondence: vquack@ukaachen.de

${ }^{\dagger} \mathrm{V}$. Quack and M. Boecker contributed equally to this work.

'Department of Orthopedic Surgery, RWTH Aachen University, Aachen,

Germany

Full list of author information is available at the end of the article
}

(c) The Author(s). 2019 Open Access This article is distributed under the terms of the Creative Commons Attribution 4.0 International License (http://creativecommons.org/licenses/by/4.0/), which permits unrestricted use, distribution, and reproduction in any medium, provided you give appropriate credit to the original author(s) and the source, provide a link to the Creative Commons license, and indicate if changes were made. The Creative Commons Public Domain Dedication waiver (http://creativecommons.org/publicdomain/zero/1.0/) applies to the data made available in this article, unless otherwise stated. 


\section{Background}

Demographic changes in the last decades led to an increased number of elderly people going along with an increase of the prevalence of degenerative disc diseases. Degenerative lumbar spinal stenosis (DLSS) is related to narrowing of the lumbar spinal canal [1] and is associated with degenerative changes of the joint complex, osteophyte formation and ligamentum flavum thickening [2]. Degeneration begins at the intervertebral disc that may cause instability of the spine segment leading to a degenerative cascade of the spine unit [2]. The prevalence of this condition, based on radiographic criteria of patients older than 60 years, is estimated to be about $50 \%$ [3]. The clinical prevalence of adults with symptoms of pain and numbness referred to the lower extremities is about 47\% [4]. Patients with DLSS may have several clinical symptoms including radiating leg pain, improvement of the pain when bending forward and gait disturbances. This often results in significant reductions in activities of daily living (ADL).

There is no "gold standard" for the diagnosis of DLSS [5]. Clinical judgement is generally based on symptom severity, functional deficits, physical examination and radiographic imaging [6]. The most promising imaging test is magnetic resonance imaging (MRI) [5]. Unfortunately, older adults often demonstrate abnormal MRI findings, even in those who are asymptomatic [7].

Only a few studies have investigated how MRI-based morphological markers are associated with symptom severity and functional deficits. Zeifang et al. found that the severity of lumbar stenosis (cross-sectional area of dural sac) and walking distance in gait analysis are not associated [8]. Likewise, Sigmundsson et al. reported a weak correlation between walking distance, pain interference with ADLs, quality of life and leg and back pain levels and the cross-sectional area of the dural sac. However, they found better general health and less leg and back pain in patients with multilevel stenosis than in patients with single-level stenosis, probably because of adaptation such that patients with a longer history of pain and limitations are more able to accept and handle the consequences of disease [9]. Kuittinen et al. compared the radiological evaluations of DLSS (classification in three severity classes based on MRI inspection of the dural sac area) with clinical findings (e.g., pain interference, pain intensity, depression, walking distance). They found no clear association between the severity of DLSS and clinical findings, but reported partly milder symptoms in patients with more severe stenosis [10]. In contrast, Hong et al. found a significant, albeit small correlation between the number of affected segments (single vs. multilevel DLSS) and pain interference [11]. Taken together, the evidence regarding the association between morphological markers and functional disability reveals small but inconsistent effects. Consequently, it remains to be elucidated which other factors influence functional disability in patients with DLSS.

It is long known that low back pain is associated with psychosocial impairment, poor sleep quality and depression [12]. Patients presenting with significant psychosocial distress at surgical evaluation have a lower quality of life and more severe physical disability. After surgery, patients with moderate psychosocial distress have poorer clinical outcomes, although, in comparison to the preoperative baseline, they improved [13]. In a 10-year follow-up after decompression surgery for lumbar stenosis, Tuomainen et al. reported that patients with lumbar stenosis with elevated depressive symptoms had an increased risk of postoperative pain and disability in the long term follow-up than patients without depressive symptoms [14].

Celestin et al. reported a systematic review of variables that predict pain-related treatment outcomes in the interventional treatment of chronic back pain. They concluded that psychological factors including somatization, depression, anxiety, and poor coping, are important predictors with greater risk of poor postoperative outcome [15]. Similarly, Lee reported associations between preoperative anxiety, optimism and postoperative patient satisfaction [16], and Ekman reported that patient factors including sex, age, preoperative working and exercise status influence postoperative outcomes [17]. Methodological factors like the use of different screening questionnaires, short followups and different indications for spine surgery make a comparison of studies a challenge.

Continuing demographic changes, increased reliance on surgery for DLSS, the increasing use of MRI imaging for DLSS diagnosis, the weak association between radiographic findings and clinical symptoms, and the influence of psychosocial factors on treatment outcome make it critical to improve our understanding of this disease pattern. Thus, the aim of this study was to evaluate the relative influence of sociodemographic characteristics, morphological markers, fear of movement as well as the degree of depression with respect to the prediction of ADL in patients with DLSS.

\section{Methods \\ Study population}

Sixty-seven in- and outpatients diagnosed with DLSS participated in this study. They were treated in a neurosurgery clinic and an orthopedic surgery clinic between May 2015 and June 2016. The data were collected as part of a larger study with patients with diverse spinal diseases which aimed at developing an instrument assessing environmental factors based on the International Classification of Functioning, Disability and Health $[18,19]$.

The primary inclusion criterion for the present study was the diagnosis of a DLSS according to the 10th revision of the International Classification of Diseases [20]. The diagnosis of DLSS was based on clinical evaluation 
by the treating spine surgeon as well as on MRI findings. Exclusion criteria were an age under 18, history of lumbar spine surgery, insufficient knowledge of the German language, severe cognitive impairment, and guardianship. The patients participated voluntarily without compensation. All participants signed an informed consent prior to participation. They completed the study questionnaires at the hospital or at home and then returned the questionnaires by mail. The study procedure was approved by the local ethics committee, RWTH Aachen University (IORG0006299), (EK026/15) and conducted according to the Declaration of Helsinki.

\section{Morphological markers}

The morphological markers were evaluated with MRI of the lumbar spine. Two of the authors (VQ and YE) evaluated the MRI images; they were blinded to the clinical symptoms and radiological report. They classified severity of lumbar stenosis according to Schizas' classification [21]: Grade A (mild), B (moderate), C (severe) or D (very severe). They documented the number of stenotic segments and presence of spondylolisthesis.

\section{Patient reported outcomes}

Patients completed the 10-item DESC-I, a Rasch-based depression screener (DESC) [22, 23]. It is a well validated screening instrument measuring depression severity on which patients describe symptom severity during the past 2 weeks using a rating scale ranging from 0 (never) to 4 (always). DESC-I scores range from 0 to 40 with higher scores indicating greater severity of depressive symptoms. A cut-off score of 12 corresponds to an interview-based diagnosis of a depressive episode according to ICD-10 criteria $[24,25]$.

Fear of movement and (re)injury was assessed using the 11-item German Version of the Tampa Scale for Kinesiophobia (TSK-GV) [26]. The TSK-GV is composed of the two subscales, TSK-somatic focus (TSK-SF, 5 items, score range: 5-20) and TSK-activity avoidance (TSK-AA, 6 items, score range: 6-24). While the TSK-SF subscale assess fear of injury in general, TSK-AA assesses avoidance of social and physical activities to minimize pain. Each item is rated on a 4-point rating scale $(1=$ "strongly disagree" to $4=$ "strongly agree") with higher scores indicating greater fear of movement and (re)injury.

ADLs were measured using a paper-pencil version of the Rasch-based RehaCAT-system [27]. RehaCAT assesses three aspects of ADL: (1) lower extremity functioning (RehaCAT-LE, e.g. walking, climbing stairs), (2) upper extremity (UE) functioning (RehaCAT-UE, e.g. self-care, eating) and (3) ADLs requiring both, upper and lower extremity functioning (RehaCAT-ADL, e.g. climbing stairs while carrying something heavy). Patients used a rating scale ranging from 0 ("without any difficulty") to 4 ("impossible") for all activities and indicated whether they were able to perform them without any help. RehaCAT-LE consists of 32 items (score range: 0128), RehaCAT-UE of 37 items (score range: 0-148) and RehaCAT-ADL of 31 items (score range: 0-124). Higher scores indicate greater disability.

The Pain Interference Scale-German (PI-G) is a 28-item scale assessing the negative effects of pain on functioning [28]. It consists of three subscales assessing pain interference with mental functioning (PI-G-mental: cognition, emotion; 13 items, score range: $0-52$ ), with recreational, household and work activities (PI-G-functional, 11 items, score range: $0-44$ ) and with physical activities like walking or standing (PI-G-physical, 4 items, score range: 0-16). PI-G items assess how much pain interfered with daily life activities during the last 7 days using a 5-point rating scale ranging from 0 ("not at all") to 4 ("very much"). Higher scores indicate greater pain interference.

The participation subscale of the Aachen Activity and Participation Index (AAPI-Part) [29] assessed the extent of participation in social, daily and work-related activities. AAPI-Part consists of 15 items which are rated on a 5point rating scale $(0=$ "I couldn't do it at all" to $4=$ "without any problems", score range: $0-60$ ). Lower scores indicate lower participation levels.

\section{Statistical analyses}

We calculated the prevalence of morphological markers (Schizas classification grades A, B, C \& D), number of stenotic segments and presence of a spondylolisthesis (yes / no). Likewise, we calculated the percentage of patients with DESC-values above the clinical cut-point. We calculated mean scale sum scores, standard deviations as well as observed sum score ranges for patient-reported outcomes (PROs) to characterize the participants.

Prior to conducting linear regression analyses, we calculated correlations between the predictor variables and the dependent variables to estimate the relationship among these variables. The predictor variables for linear regression analyses were the sociodemographic variables age and gender, duration of disease, the three morphological markers (Schizas classification, number of stenotic segments and presence of a spondylolisthesis) as well as depression (DESC) and fear of movement (TSK subscales). The dependent variables were pain interference (PI-G subscales), the performance of ADLs (RehaCAT subscales) and participation (AAPI-Part). We calculated point biserial correlations for dichotomous variables (gender and presence of a spondylolisthesis) and Pearson's correlation coefficients for continuously measured variables. Correlation coefficients ( $\mathrm{r}$ ) above |.5| were considered as evidence of strong correlations, coefficients between $|.3|$ and $|.5|$ as medium and coefficients between $|.1|$ and $|.3|$ as weak correlations. 
Subsequently, stepwise linear regression analyses were conducted to predict interference of pain with the performance of ADLs (PI-G), ADLs (RehaCAT) and participation in social life (AAPI). We included only those predictor variables which were significantly correlated with the respective outcome variable. Multicollinearity among the predictor variables was controlled using the Variance Inflation Factor (VIF). Data analysis was performed using SPSS 24. Missing data were excluded list wise.

\section{Results}

The characteristics of study participants are presented in Table 1. Study participants on average were 62.5 years old $(\mathrm{SD}=11.7)$, and $50.7 \%$ were female.

\section{Morphological markers}

Table 2 shows the clinical characteristics of the sample. Most patients experienced chronic dorsal pain as the mean duration of the disease was 6.9 years (SD: 9.2; range: 0-37 years). In over $70 \%$ of the patients more than one segment was affected by stenosis. About one third of the patients exhibited an additional spondylolisthesis. According to the Schizas classification [21] about one third of the sample was classified as moderate (B), severe (C) or extreme (D).

\section{Patient reported outcomes}

Overall, patients reported a moderate to medium level of impairment with regard to pain interference and limitations in ADLs (see Table 3). The range of reported problems and limitations varied considerably between

Table 1 Sociodemographic characteristics of 67 patients with lumbar spine stenosis

\begin{tabular}{lll}
\hline Sociodemographic Characteristics & $\%$ of patients & Mean (SD) \\
\hline Age & & \\
Gender & & \\
$\quad$ female & & \\
male & $50.7 \%$ & \\
Marital status & $49.3 \%$ & \\
married & & \\
single & $59.1 \%$ & \\
separated/divorced & $9.1 \%$ \\
living with partner & $13.6 \%$ \\
$\quad$ widowed & $6.1 \%$ \\
Current work status & $12.1 \%$ \\
employed for wages & \\
retired & $34.8 \%$ \\
disability pension & $36.4 \%$ \\
unemployed & $9.1 \%$ \\
homemaker & $7.6 \%$ \\
partial pension & $10.6 \%$ \\
\hline
\end{tabular}

Table 2 Clinical characteristics

\begin{tabular}{ll}
\hline Morphological Characteristics & $\%$ of patients \\
\hline Spondylolisthesis & $35.8 \%$ \\
Number of affected segments & \\
1 & $28.4 \%$ \\
2 & $41.8 \%$ \\
3 & $19.4 \%$ \\
4 & $10.4 \%$ \\
Schizas-classification & \\
B (moderate) & $32.8 \%$ \\
C (severe) & $35.8 \%$ \\
D (extreme) & $31.3 \%$ \\
Depression (Patient Reported Outcome) & \\
DESC $\geq 12$ & $41.5 \%)$ \\
Duration of disease & M(SD), Range \\
Duration of disease (years) & 6.9 (9.2), 0-37 \\
\hline
\end{tabular}

DESC Rasch-based depression screening

mild to severe limitations. Importantly, $41.5 \%$ of the patients had a depression score above the cut-point for depression indicating a high prevalence of clinically relevant depressive symptoms.

\section{Correlation analyses}

Table 4 shows the pattern of correlations among the predictor and dependent variables. Among the morphological markers stenosis severity was correlated significantly with the degree of activity limitations in activities requiring lower extremity functioning (RehaCAT-LE); the correlation of .32 was of medium size. The number of stenotic segments was correlated negatively albeit weakly with pain interference (PI-G mental: $r=-.28$; PI-G functional: $r=$ -.25 ) and positively but weakly with participation in daily activities (AAPI-Part; $r=.26$ ), indicating that patients with more affected segments reported less pain interference and more participation. Disease duration and performance of ADLs (RehaCAT_ADL) were correlated weakly $(\mathrm{r}=$ -.27 ). The association between the psychological predictors, depression (DESC) and fear of movement (TSK subscales) with all dependent variables were statistically significant with medium to strong effects.

Investigating the correlation pattern among the predictor variables, depression was not significantly correlated with any of the morphological markers. The same applied for fear of movement with the exception that the activity avoidance subscale (TSK-AA) correlated significantly with the severity of stenosis $(\mathrm{r}=.32, p<.01)$ indicating that more severe stenosis was associated with more activity avoidance. Depression and fear of movement were correlated significantly (DESC - TSK-AA: $\mathrm{r}=.33, \mathrm{p}<.01$; DESC - TSK-SF: $\mathrm{r}=.40, \mathrm{p}<.01)$, as were the two TSKsubscales (TSK-SF - TSK-AA: $\mathrm{r}=.54, \mathrm{p}<.01$ ). 
Table 3 Patient Reported Outcomes

\begin{tabular}{|c|c|c|c|c|c|}
\hline Patient Reported Outcomes & Assessed construct & Possible score-range & $M(S D)$ & Median & Range \\
\hline \multicolumn{6}{|l|}{ Predictor variables } \\
\hline DESC & Depression & $0-40$ & $10.9(8.6)$ & 9 & $0-35$ \\
\hline TSK & Fear of movement: & & & & \\
\hline TSK_SF & Somatic focus & $5-20$ & $10.5(3.3)$ & 10.5 & $5-19$ \\
\hline TSK_AA & Activity avoidance & $6-24$ & $14.4(4.1)$ & 14 & $6-23$ \\
\hline \multicolumn{6}{|l|}{ Dependent variables } \\
\hline $\mathrm{Pl}-\mathrm{G}$ & Pain interference: & & & & \\
\hline PI-G_mental & Mental & $0-52$ & $26.7(10.6)$ & 27 & $0-46$ \\
\hline PI-G_functional & $A D L$ & $0-44$ & $30.4(11.1)$ & 33 & $0-44$ \\
\hline PI-G_physical & Mobility & $0-16$ & $10.9(4)$ & 12 & $0-16$ \\
\hline RehaCAT & Activities of Daily Living: & & & & \\
\hline RehaCAT_LE & Lower extremity function & $0-128$ & $58.2(29.8)$ & 58.5 & $7-124$ \\
\hline RehaCAT_UE & Upper extremity function & $0-148$ & $26.5(29.2)$ & 16 & $0-122$ \\
\hline RehaCAT_ADL & ADL (UE \& LE) & $0-124$ & $69(27.6)$ & 73 & $8-123$ \\
\hline AAPI_Pa & Participation & $60-0$ & $44(12.2)$ & 47 & $60-10$ \\
\hline
\end{tabular}

DESC Rasch-based depression screening, TSK Tampa Scale for Kinesiophobia, SF Somatic focus, AA Activity avoidance, PI-G Pain Interference - German, LE Lower extremity, UE Upper extremity, $A D L$ Activities of daily living, AAPI_P Aachener Activity- and Participation Index, sub-scale participation; ${ }^{\mathrm{a}} A A P I$ only scale in which higher scores indicate better functioning

\section{Multiple linear regression analyses}

The results of the stepwise linear regression analyses are shown in Table 5. A significant proportion of variance of all dependent variables was accounted for by the predictor variables, ranging from $12 \%\left(\mathrm{R}^{2}=.12\right.$; PI-physical $)$ to $40 \%\left(R^{2}=.40\right.$; RehaCAT-LE; AAPI-P). Neither of the sociodemographic variables, age and gender, significantly contributed to the prediction of the dependent variables. Among the morphological markers only stenosis severity (Schizas-classification) contributed to the prediction of ADLs requiring LE functioning (RehaCAT-LE).

Depression and fear of movement were the most powerful predictors explaining the largest proportions of variance. Depression was the only significant predictor of pain interference with mental and physical functioning (PIG-mental; $\mathrm{R}^{2}$ change $=.32, \mathrm{~F}(1,58)=27.5, p<.01$; PIG-physical; $\mathrm{R}^{2}$ change $\left.=.12, \mathrm{~F}(1,59)=8.3, \mathrm{p}<.01\right)$, and the strongest predictor of pain interference with the performance of recreational, household and work activities (PIG-functional; $\mathrm{R}^{2}$ change $\left.=.23, \mathrm{~F}(1,59)=17.5, p<.01\right)$ and of participation in social, daily and work-related activities (AAPI-P; $\mathrm{R}^{2}$ change $=.33, \mathrm{~F}(1,56)=27, p<.01$ ). Whereas the former was additionally predicted by the activity avoidance subscale of the TSK (PIG-functional; $\mathrm{R}^{2}$ change $\left.=.05, \mathrm{~F}(1,58)=4.31, p<.05\right)$, the prediction of the latter was improved significantly by adding the somatic focus-subscale of the TSK (AAPI-P; $R^{2}$ change = $.07, \mathrm{~F}(1,55)=6.5, \mathrm{p}<.05)$. For the prediction of ADLs as assessed by the three RehaCAT-subscales, fear of movement was the strongest predictor. The somatic focus-subscale of the TSK was the only significant predictor of ADLs requiring UE functioning (RehaCAT_UE; $\mathrm{R}^{2}$ change $\left.=.40, \mathrm{~F}(1,55)=36.8, p<.01\right)$, and significantly predicted ADL requiring LE functioning (RehaCAT_LE;

Table 4 Correlations

\begin{tabular}{|c|c|c|c|c|c|c|c|c|c|}
\hline & Gender & Age & Disease Duration & Spondylolisthesis & N. of segments & Schizas-Class. & DESC & TSK_SF & TSK_AA \\
\hline PI-G_mental & .22 & -.15 & .05 & .18 & $-.28^{*}$ & -.16 & $.55^{* *}$ & $.28^{*}$ & $.25^{*}$ \\
\hline PI-G_functional & $.29^{*}$ & -.19 & -.07 & -.01 & $-.25^{*}$ & -.03 & $.48^{* *}$ & -12 & $.30^{*}$ \\
\hline PI-G_physical & .24 & -.08 & .04 & -.11 & -.04 & .06 & $.35^{* *}$ & $.26^{*}$ & .21 \\
\hline RehaCAT_LE & .20 & .11 & -.17 & -.14 & -.03 & $.32^{*}$ & $.43^{* *}$ & $.52^{* *}$ & $.45^{* *}$ \\
\hline RehaCAT_UE & -.03 & .13 & .02 & -.01 & -.04 & .23 & $.40^{* *}$ & $.64^{* *}$ & $.45^{* *}$ \\
\hline RehaCAT_ADL & $.29^{*}$ & -.06 & $-.27^{*}$ & -.18 & -.17 & .10 & $.45^{* *}$ & $.43^{* *}$ & $.44^{* *}$ \\
\hline AAPI_P & -.09 & .20 & .16 & .01 & $.26^{*}$ & -.03 & $-.57^{* *}$ & $-.39^{* *}$ & -.25 \\
\hline
\end{tabular}

N. Number, Schizas-Class. Schizas-Classification, DESC Rasch-based depression screening, TSK Tampa Scale for Kinesiophobia, SF Somatic focus, AA Activity avoidance, PI-G Pain Interference - German, LE Lower extremity, UE Upper extremity, ADL Activities of daily living, AAPI_P Aachener Activity- and Participation Index, sub-scale participation; ${ }^{*} p<.05 ;{ }^{* *} p<.01$ 
Table 5 Stepwise linear regression analyses

\begin{tabular}{|c|c|c|c|c|c|}
\hline Outcome variable & Model & Predictor variables & $R^{2}$ & $\Delta R^{2}$ & $\beta^{a}$ \\
\hline PIG_mental & 1 & DESC & .32 & $.32^{* *}$ & $.57^{* *}$ \\
\hline \multirow[t]{3}{*}{ PIG_functional } & 1 & DESC & .23 & $.23^{* *}$ & $.48^{* *}$ \\
\hline & 2 & DESC & .28 & $.05^{*}$ & $.40^{* *}$ \\
\hline & & TSK_AA & & & $.24^{*}$ \\
\hline PIG_physical & 1 & DESC & .12 & $.12^{* *}$ & $.35^{* *}$ \\
\hline \multirow[t]{6}{*}{ RehaCAT_LE } & 1 & TSK_SF & .28 & $.28^{* *}$ & $.53^{* *}$ \\
\hline & 2 & TSK_SF & .35 & $.07^{*}$ & $.50^{* *}$ \\
\hline & & Schizas-Class. & & & $.26^{*}$ \\
\hline & 3 & TSK_SF & .39 & $.04^{*}$ & $.41^{* *}$ \\
\hline & & Schizas-Class. & & & $.24^{*}$ \\
\hline & & DESC & & & $.23^{*}$ \\
\hline RehaCAT_UE & 1 & TSK_SF & .40 & $.40^{* *}$ & $.63^{* *}$ \\
\hline \multirow[t]{3}{*}{ RehaCAT_ADL } & 1 & TSK_AA & .28 & $.28^{* *}$ & $.52^{* *}$ \\
\hline & 2 & TSK_AA & .38 & $.10^{* *}$ & $.40^{* *}$ \\
\hline & & DESC & & & $.35^{* *}$ \\
\hline \multirow[t]{3}{*}{ AAPI_P } & 1 & DESC & .33 & $.33^{* *}$ & $-.57^{* *}$ \\
\hline & 2 & DESC & .40 & $.07^{*}$ & $-.45^{* *}$ \\
\hline & & TSK_SF & & & $-.29^{*}$ \\
\hline
\end{tabular}

$\beta^{a}$ : please note that the standardized $\beta$ coefficient is reported; Schizas-Class. Schizas-Classification, DESC Rasch-based depression screening, TSK Tampa Scale for Kinesiophobia, SF Somatic focus, AA Activity avoidance, PI-G Pain Interference - German, LE Lower extremity, UE Upper extremity, ADL Activities of daily living, AAPI_P Aachener Activity- and Participation Index, subscale participation; ${ }^{*} p<.05 ;{ }^{* *} p<.01$;

$\mathrm{R}^{2}$ change $\left.=.28, \mathrm{~F}(1,59)=22.9, \mathrm{p}<.01\right)$ which was also predicted by the severity of stenosis (Schizas-classification; $\mathrm{R}^{2}$ change $\left.=.07, \mathrm{~F}(1,58)=6.07, \mathrm{p}<.05\right)$ and depression $\left(\mathrm{R}^{2}\right.$ change $\left.=.04, \mathrm{~F}(1,57)=4.13, \mathrm{p}<.05\right)$. Activity avoidance and depression predicted the performance of ADLs requiring LE and UE at the same time (RehaCATADL; TSK_AA: $\mathrm{R}^{2}$ change $=.28, \mathrm{~F}(1,48)=18.2, \mathrm{p}<.01$; DESC: $\mathrm{R}^{2}$ change $\left.=.10, \mathrm{~F}(1,47)=7.9, \mathrm{p}<.01\right)$.

\section{Discussion}

The increasing number of affected patients and the growing socioeconomic consequences of DLSS underscore the need for an accurate diagnosis. Clinical judgement is generally based on symptom severity, functional deficits, physical examination and radiographic imaging [6]. However, psychological and sociodemographic factors as well as the course of disease, seem to influence the clinical presentation of DLSS (e.g. [13-15]). To understand better which factors influence the clinical presentation of DLSS, the present study investigated to what extent sociodemographic characteristics, morphological markers as well as depression and fear of movement predict limitations in ADLs and participation in social life in a sample of mostly chronic DLSS patients without a history of surgery. Results of the stepwise regression analyses revealed that pain interference with ADLs as well as limitations in ADLs and participation could be significantly predicted with the proportion of explained variance ranging between $12 \%\left(\mathrm{R}^{2}=.12\right.$; PI-Gphysical) and $40 \%\left(\mathrm{R}^{2}=.40\right.$; RehaCAT-LE; AAPI-P $)$. Neither the sociodemographic characteristics age and gender nor disease duration nor the morphological markers number of affected segments and the presence of spondylolisthesis contributed significantly to the prediction. Among the morphological markers only stenosis severity contributed to the prediction of ADLs requiring lower extremity functioning, revealing that more severe stenosis is associated with greater limitations. The variables contributing the most to the prediction of the dependent variables were the psychological factors depression and fear of movement. Depression was the strongest predictor for limitations in participation in social life as well as for pain interference with mental and physical functioning and with the execution of recreational and household activities. Fear of movement was the strongest predictor of limitations in ADLs.

The minor contribution of morphological markers in predicting ADL limitations is consistent with published findings. Most studies investigating the association between the severity of lumbar stenosis and clinical findings (e.g., pain interference, pain intensity, depression, walking distance, gait) did not report any strong correlations [8-10]. When significant correlations were found, they were generally of small size (e.g. [11], our study). Interestingly, the direction of the associations is inconsistent. In our study more severe stenosis was associated with greater limitations in ADLs requiring lower extremity functioning. Likewise, Hong et al. reported a significant, but small correlation between the number of affected segments (single vs. multilevel DLSS) and pain interference [11]. In contrast, Sigmundsson et al. reported better general health and less leg and back pain in patients with multilevel stenosis as compared to patients with single-level stenosis [9]. This finding is in agreement with our study. We found a significant, but small negative correlation between the number of stenotic segments and pain interference and a significant positive correlation with participation in daily activities. This finding suggests that under special circumstances patients with more affected segments, probably because of adaption, report less pain interference and more participation than patients with only a single affected segment. The results of Kuittinen et al.'s study point in a similar direction. They found a complex association between severity of DLSS and clinical findings, with partly milder symptoms in patients with more severe stenosis; they concluded that DLSS might not solely be an anatomical disorder, but that it has other underlying pathobiological mechanisms [10].

The literature spotlights psychological factors, as for example depression, as potential risk factors for poor clinical 
presentation (e.g., [13-15]). In our study a total of $41.5 \%$ of the patients had a clinically noticeable depression score. Furthermore, depression and fear of movement were the most powerful predictors of functional disability. The finding that fear of movement is an important predictor for limitations in ADLs and participation is in accordance with Lotzke et al.'s revised fear avoidance model (FAM) [30]. This model was developed to explain the progression of chronic musculoskeletal pain and disability [31]. According to this model, patients with a high pain-related fear, fear of movement and reduced self-efficacy are at risk of developing disabilities, depressed mood and low levels of physical activity [30]. Fear of movement as a protective reaction may be adaptive in the short term but may worsen the problem in the long term [31]. In accordance with Vlaeyen \& Linton [31], Lotzke et al. suggested identifying and targeting fear of movement preoperatively to achieve a better postoperative functional outcome [30]. Evidence in support of FAM also comes from [32] who predicted short term pain and disability following lumbar disc surgery.

Interindividual differences in reacting to pain experiences might be responsible for the inconsistent findings regarding the association between morphological markers and clinical findings. Although being indispensable for the diagnosis, morphology seems to play a minor role in explaining limitations in ADLs. Here, the way patients are able to handle the experience of pain seems to be more important as proposed by the FAM [31]. A surgeon should be aware of this influence of psychological factors when choosing the optimal intervention with regard to prognosis on quality of life after treatment.

DLSS is one of the most frequent indications for spinal surgery [33] and in literature surgical therapy, particularly recommended for severe stenosis, seems to be superior to conservative treatments [34]. Many studies show that operative treatment is associated with greater improvement of pain and function in the first years [34, 35], even so the benefits diminish over time [35]. But some patients do not benefit from surgery. Fritsch et al. conducted a metaanalysis about the clinical course of pain and disability following surgery for spinal stenosis. They report, that in general patients experienced extensive reductions in pain and disability in the first 3-month post-surgery though pain level and disability persisted at 5 years follow up [36]. Further, surgery for spinal stenosis seems to affect depression 1 year later. However, persistence of depression after surgery correlated with a worse clinical outcome and a higher rate of unmet expectations $[37,38]$. In a Cochrane database analysis, Zaina et al. found 10 to $24 \%$ complications in surgical treatment and no complications in nonoperative treatment [39]. Repeated operations may result from recurrent spinal stenosis or an increasing spondylolisthesis [40]. In a metanalysis Machado et al. reported a reoperation rate from 3 to $28 \%$ [41].
Readers should note several study limitations. While the sample size allowed us to detect small effects, it was not sufficiently large to cross validate the results. We recruited patients during in- and outpatient therapy from two clinics; results may not generalize to other clinics and regions. Because patients were free to decline study participation, selfselection bias may limit generalizability. Nonetheless, findings are consistent with prior studies and support the need to screen for and treat psychological distress before surgery.

Altogether, it is difficult but extremely important for the surgeon to identify patient factors that contribute to the understanding of who is most likely to benefit from surgery. To do so, clinical information, morphological markers and psychological variables, in particular depression and fear of movement, must be taken into account. Future studies should investigate whether psychological screening or a preoperative psychological consultation helps avoid operations or achieves better postsurgical outcomes.

\section{Conclusions}

Psychological factors may be a risk factor for a worse clinical presentation and a poorer postoperative outcome. Early psychological screening and treatment, when needed, is recommended and should be implemented routinely. This protocol may help avoid operations with a low likelihood of functional improvement and, direct treatment to patients with psychological disease who may achieve better postoperative outcomes with treatment. Further studies must show to what extent psychological screening and treatment enables better patient outcomes.

\section{Abbreviations \\ AAPI_P: Aachener Activity- and Participation Index, sub-scale participation; ADL: Activities of daily living; DESC: Rasch-based depression screening; DLSS: Degenerative lumbar spinal stenosis; LE: Lower extremity; MRI: Magnetic resonance imaging; N: Number; PI-G: Pain Interference - German; PROs: Patient-reported outcomes; r: Correlation coefficient; Schizas- Class.: Schizas-Classification; SD: Standard deviation; TSK-AA: Tampa Scale for Kinesiophobia, subscale activity avoidance; TSK-GV: Tampa Scale for Kinesiophobia; TSK-SF: Tampa Scale for Kinesiophobia, sub-scale somatic focus; UE: Upper extremity; VIF: Variance Inflation Factor}

\section{Acknowledgements \\ Not applicable for that section.}

\begin{abstract}
Authors' contributions
The contributions of the authors were as follows: VQ: conception and study design, acquisition of data, analysis and interpretation of data, manuscript writing. MBo: conception and study design, statistical analysis and data interpretation, manuscript writing. CAM: acquisition of data, substantial manuscript revision. VM: study design, interpretation of data, manuscript revision. MG: acquisition of data, substantial manuscript revision. $\mathrm{AH}$ : consultation on study design, substantial manuscript revision. MB: consultation on study design, substantial manuscript revision. YE: conception and study design, interpretation of data, substantial manuscript revision. All authors have read and approved the final manuscript.
\end{abstract}

\section{Funding}

This research project was supported by the START-program of the Faculty of Medicine, RWTH Aachen University. The funding body did not play any role 
in the design of the study, collection, analysis, and interpretation of data and in writing the manuscript.

\section{Availability of data and materials}

The datasets used and/ or analyzed during the current study are available from the corresponding author on reasonable request.

\section{Ethics approval and consent to participate}

The patients participated voluntarily without compensation. All participants signed an informed consent prior to participation. The study procedure was approved by the local ethics committee, RWTH Aachen University (IORG0006299), (EK026/15) and conducted according to the Declaration of Helsinki.

\section{Consent for publication}

Not applicable for that section.

\section{Competing interests}

The authors declare that they have no competing interests.

\section{Author details}

'Department of Orthopedic Surgery, RWTH Aachen University, Aachen, Germany. ${ }^{2}$ Department of Neurosurgery, RWTH Aachen University, Aachen, Germany. ${ }^{3}$ Department of Medical Psychology and Medical Sociology, RWTH Aachen University, Aachen, Germany. ${ }^{4}$ Department of Physical Medicine and Rehabilitation, Feinberg School of Medicine, Northwestern University, Chicago, IL, USA. ${ }^{5}$ Center for Rehabilitation Outcomes Research, Shirley Ryan AbilityLab, Chicago, IL, USA. ${ }^{6}$ The Orthopedic Department, Alexandria University, Alexandria, Egypt.

\section{Received: 18 April 2019 Accepted: 29 October 2019}

Published online: 23 November 2019

\section{References}

1. Genevay S, Atlas SJ. Lumbar spinal stenosis. Best Pract Res Clin Rheumatol. 2010;24:253-65.

2. Abbas J, Slon V, Stein D, Peled N, Hershkovitz I, Hamoud K. In the quest for degenerative lumbar spinal stenosis etiology: the Schmorl's nodes model. BMC Musculoskelet Disord. 2017;18(1):164.

3. Kalichman L, Cole R, Kim DH, Li L, Suri P, Guermazi A, et al. Spinal stenosis prevalence and association with symptoms: the Framingham study. Spine J. 2009;9:545-50.

4. Konno S, Hayashino Y, Fukuhara S, Kikuchi S, Kaneda K, Seichi A, et al. Development of a clinical diagnosis support tool to identify patients with lumbar spinal stenosis. Eur Spine J. 2007:16(11):1951-7.

5. De Schepper EL, Overdevest GM, Suri P, Peul WC, Oei EH, Koes BW, et al. Diagnosis of lumbar spinal stenosis: et al an updated systematic review of the accuracy of diagnostic tests. Spine (Phila Pa 1976). 2013;38(8):E 469-81.

6. Karp JF, McGovern J, Marron MM, Gerszten P, Weiner DK, Okonkwo D, et al. Clinical and neuropsychiatric correlates of lumbar spinal surgery in older adults: results of a pilot study. Pain Manag. 2016;6(6):543-52.

7. Boden SD, Davis DO, Dina TS, Patronas N, Wiesel S. Abnormal magneticresonance scans of the lumbar spine in asymptomatic subjects. A prospective investigation. J Bone Joint Surg Am. 1990;72:403-8.

8. Zeifang F, Schiltenwolf M, Abel R, Moradi B. Gait analysis does not correlate with clinical and MR imaging parameters in patients with symptomatic lumbar spinal stenosis. BMC Musculoskelet Disord. 2008;9:89.

9. Sigmundsson FG, Kang XP, Jönsson B, Strömqvist B. Correlation between disability and MRI findings in lumbar spinal stenosis. A prospective study of 109 patients operated on by decompression. Acta Orthop. 2011;82(2):204-10.

10. Kuittinen P, Sipola P, Saari T, Aalto TJ, Sinikallio S, Savolainen S, et al. Visually assessed severity of lumbar spinal canal stenosis is paradoxically associated with leg pain and objective walking ability. BMC Musculoskelet Disord. 2014;15:348.

11. Hong JH, Lee MY, Jung SW, Lee SY. A significant correlation was shown between those patients with multilevel LSS and the ODI scores; however, significant correlations were not found between the MRI findings and the psychological factors pertaining to sleep and life qualities. Korean J Anesthesiol. 2015:68(5):481-7.

12. Ferrell BA. Pain management in elderly people. J Am Geriatr Soc. 1991;39(1):64-73.
13. Amaral V, Marchi L, Martim H, Amaral R, Nogueira-Neto J, Pierro E, et al. Influence of psychosocial distress in the results of elective lumbar spine surgery. J Spine Surg. 2017;3(3):371-8.

14. Tuomainen I, Pakarinen $M$, Aalto $T$, Sinikallio $\mathrm{S}$, Kröger $\mathrm{H}$, Viinamäki $\mathrm{H}$, et al. Depression is associated with the long-term outcome of lumbar spinal stenosis surgery: a 10-year follow-up study. Spine J. 2018;18(3):458-63.

15. Celestin J, Edwards RR, Jamison RN. Pretreatment psychosocial variables as predictors of outcomes following lumbar surgery and spinal cord stimulation: a systematic review and literature synthesis. Pain Med. 2009;10:639-53.

16. Lee J, Kim HS, Shim KD, Park YS. The effect of anxiety, depression, and optimism on postoperative satisfaction and clinical outcomes in lumbar spinal stenosis and degenerative spondylolisthesis patients: cohort study. Clin Orthop Surg. 2017:9(2):177-83.

17. Ekman $\mathrm{P}$, Möller $\mathrm{H}$, Hedlund R. Predictive factors for the outcome of fusion in adult isthmic spondylolisthesis. Spine (Phila Pa 1976). 2009:34(11):1204-10.

18. World Health Organization. International classification of functioning, disability and health. Geneva: World Health Organization; 2001.

19. Gecht J, Mainz V, Boecker M, Clusmann H, Geiger MF, Tingart M, et al. Development of a short scale for assessing economic environmental aspects in patients with spinal diseases using Rasch analysis. Health Qual Life Outcomes. 2017;15(1):196.

20. International Statistical Classification of Diseases and related health problems, 10th Revision; 2016. http//:icd.who.int/browse10/2016/en.

21. Schizas C, Theumann N, Burn A, Tansey R, Wardlaw D, Smith FW, et al. Qualitative grading of severity of lumbar spinal stenosis based on the morphology of the dural sac on magnetic resonance images. Spine (Phila Pa 1976). 2010;35(21):1919-24.

22. Forkmann T, Norra C, Wirtz M, Gauggel S, Boecker M. Psychometric evaluation of the Rasch-based depression screening in patients with neurologic disorders. Arch Phys Med Rehabil. 2010;91(8):1188-93.

23. Forkmann T, Boecker M, Wirtz M, Glaesmer H, Brähler E, Norra C, et al. Validation of the Rasch-based depression screening in a large scale German general population sample. Health Qual Life Outcomes. 2010;8:105.

24. Forkmann $T$, Boecker $M$, Wirtz M, Eberle $N$, Westhofen $M$, Schauerte $P$, et al. Development and validation of the Rasch-based depression screening (DESC) using Rasch analysis and structural equation modelling. J Behav Ther Exp Psychiatry. 2009;40(3):468-78.

25. Vehren T, Boecker M, Norra C, Wirtz M, Gauggel S, Forkmann T. Crosssectional validation of the Rasch-based depression screening (DESC) in a mixed sample of patients with mental and somatic diseases. Compr Psychiatry. 2013;54(7):1082-9.

26. Rusu AC, Kreddig N, Hallner D, Hülsebusch J, Hasenbring M. Fear of movement/ (re)injury in low back pain: confirmatory validation of a German version of the Tampa scale for Kinesiophobia. BMC Musculoskelet Disord. 2014;15:280.

27. Scholz M, Müller E, Kröhne U, Böcker M, Forkmann T, Wirtz M. Validierung der computeradaptiven Assessmentprozedur RehaCAT hinsichtlich funktionaler und psychischer Belastungen bei Rehabilitanden mit muskuloskelettalen Erkrankungen. [validation of the computerized adaptive assessment procedure RehaCAT related to functional and mental strain in a sample of rehabilitation patient suffering from musculoskeletal diseases]. Rehabilitation. 2014:53:290-6.

28. Farin E, Nagl M, Gramm L, Heyduck K, Glattacker M. Development and evaluation of the PI-G: a three-scale measure based on the German translation of the PROMIS_ pain interference item bank. Qual Life Res. 2014; 23:1255-65.

29. Boecker M, Elhan A, Tennant A, Wirtz M, Eberle N, Gauggel S. On the way to the NeuroCAT: development and initial evaluation of the Aachen-ADLItembank. Copenhagen: Rasch Conference; 2010. p. 13-6.

30. Lotzke H, Jakobsson M, Gutke A, Hagströmer M, Brisby H, Hägg O, et al. Patients with severe low back pain exhibit a low level of physical activity before lumbar fusion surgery: a cross-sectional study. BMC Musculoskelet Disord. 2018:19(1):365.

31. Vlaeyen JW, Linton SJ. Fear-avoidance model of chronic musculoskeletal pain: 12 years on. Pain. 2012:153(6):1144-7.

32. Alodaibi FA, Fritz JM, Thackeray A, Koppenhaver SL, Hebert JJ. The fear avoidance model predicts short-term pain and disability following lumbar disc surgery. PLoS One. 2018;13(3):e0193566.

33. Deyo RA. Treatment of lumbar spinal stenosis: a balancing act. Spine J. 2010;10:625-7.

34. Weinstein JN, Tosteson TD, Lurie JD, Tosteson A, Blood E, Herkowitz H, et al. Surgical versus nonoperative treatment for lumbar spinal stenosis four-year 
results of the spine patient outcomes research trial. Spine (Phila Pa 1976). 2010;35:1329-38

35. Lurie JD, Tosteson TD, Tosteson A, Abdu W, Zhao W, Morgan TS, et al. Long-term outcomes of lumbar spinal stenosis: eight-year results of the spine patient outcomes research trial (SPORT). Spine (Phila Pa 1976). 2015; 40(2):63-76.

36. Fritsch CG, Ferreira ML, Maher CG, Herbert RD, Pinto RZ, Koes B, et al. The clinical course of pain and disability following surgery for spinal stenosis: a systematic review and meta-analysis of cohort studies. Eur Spine J. 2017; 26(2):324-35.

37. Falavigna A, Righesso O, Teles AR, Conzati LP, Bossardi JB, da Silva PG, et al. Responsiveness of depression and its influence on surgical outcomes of lumbar degenerative diseases. Eur J Orthop Surg Traumatol. 2015;25(Suppl 1):S35-41.

38. Urban-Baeza A, Zárate-Kalfópulos B, Romero-Vargas S, Obil-Chavarría C, Brenes-Rojas L, Reyes-Sánchez A. Influence of depression symptoms on patient expectations and clinical outcomes in the surgical management of spinal stenosis. J Neurosurg Spine. 2015;22(1):75-9.

39. Zaina F, Tomkins-Lane C, Carragee E, Negrini S. Surgical versus non-surgical treatment for lumbal spinal stenosis (review). Cochrane Database Syst Rev. 2016;1:CD010264.

40. Gerling MC, Leven D, Passias PG, Lafage V, Bianco K, Lee A, et al. Risk factors for reoperation in patients treated surgically for lumbar stenosis: a subanalysis of the 8 year data from the SPORT trial. Spine (Phila Pa 1976). 2016;41(10):901-9.

41. Machado G, Ferreira P, Harris I, Pinheiro M, Koes B, van Tulder M, et al. Effectiveness of surgery for lumbar spinal stenosis: a systematic review and metaanalysis. PLoS One. 2015;10(3):e0122800.

\section{Publisher's Note}

Springer Nature remains neutral with regard to jurisdictional claims in published maps and institutional affiliations.

Ready to submit your research? Choose BMC and benefit from:

- fast, convenient online submission

- thorough peer review by experienced researchers in your field

- rapid publication on acceptance

- support for research data, including large and complex data types

- gold Open Access which fosters wider collaboration and increased citations

- maximum visibility for your research: over $100 \mathrm{M}$ website views per year

At $\mathrm{BMC}$, research is always in progress.

Learn more biomedcentral.com/submissions 